\title{
A fixed point conjecture for Borsuk continuous set-valued mappings
}

by

\author{
Dariusz Miklaszewski (Toruń)
}

\begin{abstract}
The main result of this paper is that for $n=3,4,5$ and $k=n-2$, every Borsuk continuous set-valued map of the closed ball in the $n$-dimensional Euclidean space with values which are one-point sets or sets homeomorphic to the $k$-sphere has a fixed point. Our approach fails for $(k, n)=(1,4)$. A relevant counterexample (for the homological method, not for the fixed point conjecture) is indicated.
\end{abstract}

1. Introduction. The Lefschetz fixed point theorem holds for uppersemicontinuous mappings with acyclic values and for their compositions [8], [11]. On the other hand, even the Hausdorff continuity does not guarantee an extension of the Brouwer theorem when the values are spheres [23]. Nevertheless, one can expect some fixed point results for mappings with nonacyclic values provided a stronger kind of continuity is assumed. In 1954 Borsuk defined a distance $\varrho_{\mathrm{c}}$ in the hyperspace $K(M)$ of all nonempty compact subsets of a metric space $(M, \varrho)$ and called it the metric of continuity [1]. Let us recall that $\varrho_{\mathrm{c}}(X, Y)=\max \left\{d_{\mathrm{c}}(X, Y), d_{\mathrm{c}}(Y, X)\right\}$, where $d_{\mathrm{c}}(X, Y)=\inf \{\max \{\varrho(x, g(x)): x \in X\}\}$ and the infimum is taken over all continuous functions $g$ from $X$ to $Y$. We call a map into $K(M)$ Borsuk continuous if it is continuous with respect to $\varrho_{\mathrm{c}}$. Let $B^{n}$ denote the closed unit ball in $\mathbb{R}^{n}$. Górniewicz posed the following conjecture $\left({ }^{1}\right)$ :

(G.C.) Every Borsuk continuous map $f: B^{n} \rightarrow K\left(B^{n}\right)$ with connected values has a fixed point $x \in f(x)$.

2000 Mathematics Subject Classification: 54C60, 55M20, 55R25, 57R20.

Key words and phrases: set-valued mapping, fixed point, Borsuk continuity, bundle, characteristic class.

$\left({ }^{1}\right)$ Górniewicz published this conjecture in $[13,5.6]$ as an open problem, long after communicating it to students and proving its two-dimensional case. It was called the Górniewicz Conjecture in [21], in honour of Górniewicz's 60th birthday. The proof for $n=2$ is based on [12] and can be found in [4]. 
In this paper we study a special case of G.C.:

Conjecture 1. Every Borsuk continuous map $f: B^{n} \rightarrow K\left(B^{n}\right)$ with values which are one-point sets or sets homeomorphic to the sphere $S^{k}$ $(k$ fixed) has a fixed point.

We will denote by $*$ the one-point space. The G.C. is confirmed for maps $f$ with the rational Čech cohomology group $\check{H}^{\star}(f(x) ; \mathbb{Q})$ isomorphic to $\check{H}^{\star}(* ; \mathbb{Q})$ or to $\check{H}^{\star}\left(S^{n-1} ; \mathbb{Q}\right)$ for $x \in B^{n}$ (see [13]). The latter case clearly implies Conjecture 1 for $k=n-1$. The proof is based on the fact that the set $\widetilde{f}(x)=f(x) \cup b\left(\mathbb{R}^{n} \backslash f(x)\right)$ is acyclic, where $b\left(\mathbb{R}^{n} \backslash f(x)\right)$ denotes the bounded component of $\mathbb{R}^{n} \backslash f(x)$. Since Cech homology spheres of codimension greater than 1 do not separate $\mathbb{R}^{n}$, it is clear that this approach cannot work for $1 \leq k \leq n-2\left({ }^{2}\right)$. Our purpose is to prove Conjecture 1 for $k=n-2$ and $n=3,4,5$. A different proof of the $n=3$ case was given in [21]. In Preliminaries we give a brief exposition of some results from [20], which are basic for this paper.

2. Preliminaries. Let $f: B^{n} \rightarrow K\left(B^{n}\right)$ be an upper-semicontinuous map. From now on, $B=B^{n}$ and $S=\partial B$. For any $C \subset B$ we will denote by $\Gamma_{C}$ the set $\{(x, y) \in C \times B: y \in f(x)\}$, called the graph of $\left.f\right|_{C}$. Let $F$ be a field.

Definition 1 ([20]). The map $f$ is called an F-Brouwer map if the homomorphism

$$
\check{H}_{n}\left(\Gamma_{B}, \Gamma_{S} ; F\right) \rightarrow \check{H}_{n}(B \times B, S \times B ; F)
$$

induced by inclusion is a nonzero homomorphism.

Theorem 1 [20, Lemma 1]. Every F-Brouwer map has a fixed point.

Let $f$ satisfy the hypotheses of Conjecture 1 and define $U=\{x \in B$ : $\left.f(x) \cong S^{k}\right\}$. By the Chapman-Ferry-Jakobsche results on approximating homotopy equivalences by homeomorphisms [9, Theorem 3], [2, $\alpha$-approximation theorem], [16], [17] for $k \neq 4\left({ }^{3}\right),\left.f\right|_{U}$ is continuous with respect to the distance $\varrho_{\mathrm{h}}$ which is defined similarly to $d_{\mathrm{c}}$, but the infimum is taken over all homeomorphisms $g$ from $X$ onto $Y$. This continuity implies that the projection $p: \Gamma_{U} \rightarrow U$ is a completely regular mapping [6]. Consequently, $p$ is a locally trivial bundle with fibre $S^{k}$ (see [6]; cf. also [3, Theorem, p. 131] and $[7$, Corollary 1.1, p. 63]).

$\left({ }^{2}\right)$ Though $S^{0}$ is not connected, Conjecture 1 holds for $k=0$. In this case $f$ may be identified with a continuous mapping into the second symmetric product of its domain. The fixed point theory of such mappings is developed in [25], [26], [22]. In spite of these results the G.C. cannot be extended to maps with disconnected values [18].

$\left({ }^{3}\right)$ The author does not know if the $\alpha$-approximation theorem holds in dimension 4 . 
Definition 2. We say that an $(n-1)$-dimensional topological manifold $N$ e-approximates $\partial U$ in $U$ if there exists a compact $n$-dimensional topological manifold $K$ with boundary such that $K$ is a subcomplex of a simplicial decomposition of $B, \partial K=N$ and $U \supset K \supset U \backslash O_{\varepsilon}(\partial U)$, where $O_{\varepsilon}(\partial U)=\{x \in U: \operatorname{dist}(x, \partial U)<\varepsilon\}$.

We begin with a triangulation of the interior of $B$ with mesh $\leq \varepsilon / 2$. Then let $K^{\prime}$ be the union of all simplices intersecting $U \backslash O_{\varepsilon}(\partial U)$ and let $K$ be a small regular neighbourhood of $K^{\prime}$. It is clear that $\partial K \varepsilon$-approximates $\partial U$ in $U$. We can now rephrase [20, Theorem 1] as follows:

THEOREM 2. Suppose that:

1. $f: B \rightarrow K(B)$ is a Borsuk continuous map,

2. $f$ is singlevalued on $B \backslash U$ and takes values homeomorphic to $S^{k}$ on $U, k \neq 4$,

3. $U \subset \operatorname{Int} B\left({ }^{4}\right)$,

4. For every $\varepsilon>0$ there exists a manifold $N$ which $\varepsilon$-approximates $\partial U$ in $U$ and satisfies the inequality

$$
\operatorname{dim} H_{k}\left(\Gamma_{M} ; \mathbb{Z}_{2}\right)>\operatorname{dim} H_{k}\left(M ; \mathbb{Z}_{2}\right)
$$

for all components $M$ of $N$.

Then $f$ is a $\mathbb{Z}_{2}$-Brouwer map.

This theorem follows directly from [20, proof of Theorem 1] and generalizations of $[15,3.4 .3,3.4 .6]$ to $\operatorname{TOP}\left(S^{k}\right)$-bundles, where $\operatorname{TOP}\left(S^{k}\right)$ denotes the group of all homeomorphisms $S^{k} \rightarrow S^{k}$.

3. A conjecture on homology of sphere bundles. It is of interest to know when assumption 4 of Theorem 2 is satisfied. Since the projection from $\Gamma_{U}$ onto $U$ is a locally trivial bundle with fibre $S^{k}$, so is $p: \Gamma_{M} \rightarrow M$. In fact, $p$ may be considered as a bundle with structural group $\operatorname{TOP}\left(S^{k}\right)$. Moreover, the diagram

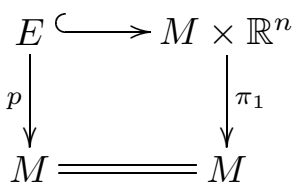

commutes for $E=\Gamma_{M}$. In Section 6 we will prove the following

$\left({ }^{4}\right)$ If $f$ is not singlevalued on $S$, we extend it to $2 B$ taking $\widetilde{f}(x)=(2-\|x\|) f\left(\|x\|^{-1} x\right)$ for $1 \leq\|x\| \leq 2$. Of course, $\widetilde{f}$ is singlevalued on $2 S$. Moreover, by [20, proof of Statement 6$]$, if $\tilde{f}$ is a $\mathbb{Z}_{2}$-Brouwer map, so is $f$. It would be nice to have Theorem 2 without the third assumption. Unfortunately, the author does not know if the fourth hypothesis for $f$ implies the same for $\tilde{f}$. 
TheOREM 3. Let $M \subset \mathbb{R}^{n}$ be a compact connected $(n-1)$-dimensional topological manifold without boundary, $n \geq 3$. Let $p: E \rightarrow M$ be a bundle with fibre $S^{n-2}$ and structural group $O(n-1)$ such that the diagram $(\sharp)$ commutes. Then

$$
\operatorname{dim} H_{n-2}\left(E ; \mathbb{Z}_{2}\right)>\operatorname{dim} H_{n-2}\left(M ; \mathbb{Z}_{2}\right) .
$$

REMARK 1. It suffices to assume in Theorem 3 that the structural group reduces to $O(n-1)$.

Recall that the structural group $G$ reduces to the subgroup $H$ if every bundle $p: E \rightarrow M$ with structural group $G$ is $G$-equivalent to a bundle $\widetilde{p}$ : $\widetilde{E} \rightarrow M$ with structural group $H$ (in particular, there is a homeomorphism $h: \widetilde{E} \rightarrow E$ with $p \circ h=\widetilde{p})$. Moreover, if the inclusion $O(n-1) \subset G$ is a homotopy equivalence, then $G$ reduces to $O(n-1)$ [30, proof of 11.45].

Conjecture 2. Theorem 3 remains true with $O(n-1)$ replaced by $\operatorname{TOP}\left(S^{n-2}\right)$.

FACT 1. Conjecture 2 holds for $n \in\{3,4,5\}$.

Fact 1 follows from the homotopy equivalences $O(2) \simeq \operatorname{TOP}\left(S^{1}\right)$ (see for instance [20, Fact 2]), $O(3) \simeq \operatorname{TOP}\left(S^{2}\right)\left([19]\right.$, see also [28]), $O(4) \simeq \operatorname{TOP}\left(S^{3}\right)$ $[14$, p. 606].

4. A fixed point theorem. This section contains the main result of this paper. According to Fact 1 and Theorem 1-3, we have the following

Theorem 4. Conjecture 1 is true for $(k, n)=(1,3),(2,4),(3,5)$.

The case $(k, n)=(1,3)$ (first proved in [21] using $K$-theory) has a nice geometric interpretation: the Brouwer fixed point theorem holds for Borsuk continuous maps whose values are points or knots. One thing that distinguishes the case $(k, n)=(1,3)$ from other cases is the classification of all 1-sphere bundles over 2-manifolds up to weak bundle equivalence [27] (see also [24], [10]).

\section{Preparation for proving Theorem 3}

Lemma 1. Let $M \subset \mathbb{R}^{n+1}$ be an $n$-dimensional compact connected topological manifold without boundary, $n \geq 2$. Then $x^{n}=0$ for every $x \in$ $H^{1}\left(M ; \mathbb{Z}_{2}\right)$. Here $x^{n}$ denotes the cup product $x^{n-1} \cup x$.

The situation described in the hypotheses of this lemma is very well known in the literature. Let us gather some facts before the proof. First, $M \subset \mathbb{R}^{n+1} \cup\{\infty\} \cong S^{n+1}$ and $S^{n+1} \backslash M=U \cup V$ (U,V connected). The closures $A=\bar{U}, B=\bar{V}$ are ANR's [5, VIII.4.8]. By the Alexander duality, $H^{n}\left(A ; \mathbb{Z}_{2}\right)=H^{n}\left(B ; \mathbb{Z}_{2}\right)=0$. Let $i: M \rightarrow A$ and $j: M \rightarrow B$ be inclusions. 
The Mayer-Vietoris exact sequence shows that $\varphi: H^{s}\left(A ; \mathbb{Z}_{2}\right) \oplus H^{s}\left(B ; \mathbb{Z}_{2}\right) \rightarrow$ $H^{s}\left(M ; \mathbb{Z}_{2}\right), \varphi(\alpha, \beta)=i^{\star} \alpha+j^{\star} \beta$, is an isomorphism for $1 \leq s<n$. Moreover:

(1) $\mathrm{Sq}^{n-r} y=0$ for every $y \in H^{r}\left(M ; \mathbb{Z}_{2}\right), 1 \leq r<n$ [29, III.2.3];

(2) $i^{\star} \mathrm{Sq}^{1} a \cup j^{\star} b=i^{\star} a \cup j^{\star} \mathrm{Sq}^{1} b$ for all $a \in H^{r}\left(A ; \mathbb{Z}_{2}\right), b \in H^{n-1-r}\left(B ; \mathbb{Z}_{2}\right)$ ([29, III.2.4], see also [29, II.4, III.1.4]);

(3) $\mathrm{Sq}^{i} u^{k}=\left(\begin{array}{c}k \\ i\end{array}\right) u^{k+i}$ if $\operatorname{dim}(u)=1$ [29, I.2.4].

Proof of Lemma 1. Case 1. Let $n \neq 2^{m}-1$ for every natural $m$. Since $0=\mathrm{Sq}^{n-r} x^{r}=\left(\begin{array}{c}r \\ n-r\end{array}\right) x^{n}$ by (1), (3), it suffices to find $r$ such that $\left(\begin{array}{c}r \\ n-r\end{array}\right)$ is odd and $1 \leq n-r \leq r<n$. If $n=2 t$ then $r=t$ satisfies the above conditions. If $n=2 t-1$ then $t \neq 2^{m-1}$ for every $m$. Thus $t=2^{i-1}+j$ for some $i \geq 2$ and $1 \leq j \leq 2^{i-1}-1$. It is easy to check that $\left(\begin{array}{c}2^{i}-1 \\ k\end{array}\right)$ is odd $\left({ }^{5}\right)$ for every $k=0,1, \ldots, 2^{i}-1$ and $r=2^{i}-1$ satisfies $1<n-r<r<n$.

Case 2. Let $n=2^{m}-1$. Then

$$
\begin{aligned}
x^{n} & =\left(i^{\star} \alpha+j^{\star} \beta\right)^{n}=\sum_{k=0}^{n}\left(\begin{array}{l}
n \\
k
\end{array}\right) i^{\star} \alpha^{k} \cup j^{\star} \beta^{n-k}=\sum_{k=1}^{n-1} i^{\star} \alpha^{k} \cup j^{\star} \beta^{n-k} \\
& =\sum_{p=1}^{(n-1) / 2}\left(i^{\star} \alpha^{2 p-1} \cup j^{\star} \beta^{n-2 p+1}+i^{\star} \alpha^{2 p} \cup j^{\star} \beta^{n-2 p}\right) \\
& =\sum_{p=1}^{(n-1) / 2}\left(i^{\star} \alpha^{2 p-1} \cup j^{\star}\left(\begin{array}{c}
n-2 p \\
1
\end{array}\right) \beta^{n-2 p+1}+i^{\star}\left(\begin{array}{c}
2 p-1 \\
1
\end{array}\right) \alpha^{2 p} \cup j^{\star} \beta^{n-2 p}\right) \\
& =\sum_{p=1}^{(n-1) / 2}\left(i^{\star} \alpha^{2 p-1} \cup j^{\star} \mathrm{Sq}^{1} \beta^{n-2 p}+i^{\star} \mathrm{Sq}^{1} \alpha^{2 p-1} \cup j^{\star} \beta^{n-2 p}\right)=0,
\end{aligned}
$$

by (2), which proves the lemma.

We now recall some properties of Stiefel-Whitney classes. The first fact generalizes the well known Borsuk-Ulam theorem:

FACT 2. Let $E$ be a compact space, $T: E \rightarrow E$ a fixed point free involution (or equivalently, the generator of a free $\mathbb{Z}_{2}$-action on $E$ ), $c \in$ $H^{1}\left(E / T ; \mathbb{Z}_{2}\right)$ the first Stiefel-Whitney class of the 0-sphere bundle $\pi: E \rightarrow$ $E / T$ and $g: E \rightarrow \mathbb{R}^{n}$ a continuous function. Suppose that $c^{n} \neq 0$. Then there is $x \in E$ such that $g(x)=g(T x)$.

We give no reference here, because Fact 2 is an immediate consequence of the naturality of Stiefel-Whitney classes. Now, let $p: E \rightarrow M$ be a $k$-sphere bundle with structural group $O(k+1)$. The antipodal map of $S^{k}$ induces a

$\left(^{5}\right)$ By induction on $i,(x+y)^{q} \bmod 2=x^{q}+y^{q}$ for $q=2^{i}$, so $\left(\begin{array}{c}2^{i} \\ k\end{array}\right)$ is even for $k=1, \ldots, 2^{i}-1$. 
fibre preserving fixed point free involution $T: E \rightarrow E, p \circ T=p$. We have a projection $q: E / T \rightarrow M$ with $q \circ \pi=p(\pi, c$ are defined in Fact 2).

FACT $3[15,16.2 .5]$. The group $H^{\star}\left(E / T ; \mathbb{Z}_{2}\right)$ is an $H^{\star}\left(M ; \mathbb{Z}_{2}\right)$-module freely generated by $\left\{1, c, \ldots, c^{k}\right\}$ with the multiplication

$$
H^{\star}\left(M ; \mathbb{Z}_{2}\right) \times H^{\star}\left(E / T ; \mathbb{Z}_{2}\right) \ni(\alpha, \beta) \mapsto \alpha \beta=q^{\star}(\alpha) \cup \beta .
$$

Moreover, $c^{k+1}=\sum_{j=1}^{k+1} w_{j} c^{k+1-j}$, where $w_{j}$ are the Stiefel-Whitney classes of the bundle $p$.

FACT $4\left[21\right.$, Lemma 1]. Let $M$ be a compact $A N R$. Then $\operatorname{dim} H_{k}\left(E ; \mathbb{Z}_{2}\right)$ $>\operatorname{dim} H_{k}\left(M ; \mathbb{Z}_{2}\right)$ if and only if $w_{k+1}=0$.

6. Proof of Theorem 3. We begin by extending the diagram ( $\sharp)$ :

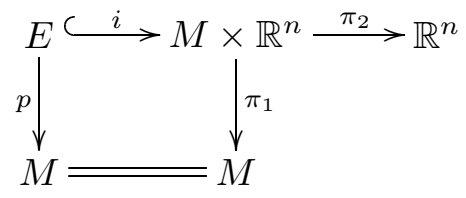

Suppose that $c^{n} \neq 0$. From Fact 2 with $g=\pi_{2} \circ i$, we obtain points $x$ and $y=T(x)$ such that $\pi_{2} \circ i(x)=\pi_{2} \circ i(y)$. Since $\pi_{1} \circ i(y)=p(y)=$ $p(x)=\pi_{1} \circ i(x)$, it follows that $i(x)=i(y)$ and $x=y$, which contradicts the fact that $T$ is fixed point free. Thus $c^{n}=0$. Fact 3 for $k=n-2$ leads to $c^{n-1}=\sum_{j=1}^{n-1} w_{j} c^{n-1-j}$. Hence

$$
\begin{aligned}
0 & =c^{n-1} \cup c=\sum_{j=1}^{n-1} w_{j} c^{n-j}=w_{1} c^{n-1}+\sum_{j=2}^{n-1} w_{j} c^{n-j} \\
& =w_{1} \sum_{j=1}^{n-1} w_{j} c^{n-1-j}+\sum_{j=1}^{n-2} w_{j+1} c^{n-j-1} \\
& =\left(w_{1} \cup w_{n-1}\right) 1+\sum_{j=1}^{n-2}\left(w_{1} \cup w_{j}+w_{j+1}\right) c^{n-j-1} .
\end{aligned}
$$

By Fact $3, w_{1} \cup w_{j}+w_{j+1}=0$ for $j=1, \ldots, n-2$. This gives $w_{n-1}=$ $\left[w_{1}\right]^{n-1}=0$, by Lemma 1 . Fact 4 completes the proof.

The same proof with Fact 2 applied to $g=\pi_{2} \circ i \circ h(h: \widetilde{E} \rightarrow E$ a bundle equivalence) yields Remark 1.

5. A counterexample. In this section it is shown that the notion of $F$ Brouwer mapping is not suitable for proving Conjecture 1 for $(k, n)=(1,4)$. It is worth pointing out that our example does have an obvious fixed point. 
THEOREM 5. There is a Borsuk continuous mapping $f: B^{4} \rightarrow K\left(B^{4}\right)$ with values homeomorphic to $*$ or $S^{1}$, which is an F-Brouwer map for no field $F$.

Proof. Part 1. Write $x=\sum_{i=1}^{4} x_{i} e_{i} \in \mathbb{R}^{4},\left(e_{i}\right)_{i=1}^{4}$ the standard basis in $\mathbb{R}^{4}, \mathbb{R}^{i}=\operatorname{span}\left\{e_{1}, \ldots, e_{i}\right\}$ for $i \leq 4, S^{3}$ the unit sphere in $\mathbb{R}^{4}, S^{i-1}=S^{3} \cap \mathbb{R}^{i}$, $E_{x}=\operatorname{span}\left\{x, e_{3}, e_{4}\right\}$ for $x \in S^{1}, S_{x}=S^{3} \cap E_{x}$. Define $\varphi_{x}: S^{2} \rightarrow S_{x}$ by $\varphi_{x}(y)=y_{1} x+y_{2} e_{3}+y_{3} e_{4}$ for $x \in S^{1}$ and $\varphi: S^{1} \times S^{2} \rightarrow \mathbb{R}^{4}$ by $\varphi(x, y)=\frac{1}{2} x+\frac{1}{4} \varphi_{x}(y)$. The map $\varphi$ is an embedding of $S^{1} \times S^{2}$ in $\mathbb{R}^{4}$. Set

$$
K=\left\{\frac{1}{2} x+r \cdot \frac{1}{4} \varphi_{x}(y):(x, y) \in S^{1} \times S^{2}, 0 \leq r \leq 1\right\} .
$$

Clearly, $K \cong S^{1} \times B^{3}$. Let $q: S^{3} \rightarrow S^{2}$ be the Hopf fibration. We define $f: B^{4} \rightarrow K\left(B^{4}\right)$ by the formula

$$
\begin{cases}f\left(\frac{1}{2} x+r \cdot \frac{1}{4} \varphi_{x}(y)\right)=r(1-r) \cdot q^{-1}(y) & \text { on } K, \\ f(z)=0 & \text { on } B^{4} \backslash K .\end{cases}
$$

Part 2. Suppose, contrary to our claim, that there is a field $F$ making $f$ an $F$-Brouwer map. Set $B=B^{4}, S=\partial B$ and $H_{\star}(\cdot)=H_{\star}(\cdot ; F)$. Note that $\left.f\right|_{S}=0$. The commutative diagram



with $j(x)=(x, 0)$ yields $p_{\star} \neq 0$. The diagram

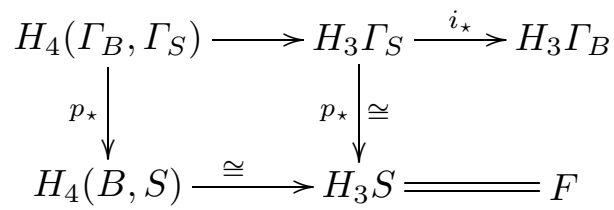

with the first row exact shows that $i_{\star}=0$. Let $C=B \backslash \operatorname{Int}(K)$. Consider the segment of the Mayer-Vietoris exact sequence:

$$
H_{4} B \rightarrow H_{3}(\partial K) \rightarrow H_{3} C \oplus H_{3} K \rightarrow H_{3} B .
$$

Since $H_{3} K=H_{3}\left(S^{1} \times B^{3}\right)=0$ and $H_{3}(\partial K)=H_{3}\left(S^{1} \times S^{2}\right)=F$, we have $H_{3} C=F$. Take $v \in \operatorname{Int}(K)$. Since $S$ is a strong deformation retract of $B \backslash\{v\}$, the composition

$$
F=H_{3} S \stackrel{\eta}{\rightarrow} H_{3} C \rightarrow H_{3}(B \backslash\{v\})
$$

of homomorphisms induced by inclusions is an isomorphism. Therefore $\eta$ is a monomorphism. Now, the equality $H_{3} C=F$ shows that $\eta$ is an isomorphism. Since $\Gamma_{S}=S \times 0$ and $\Gamma_{C}=C \times 0$, also $\bar{\eta}: H_{3} \Gamma_{S} \rightarrow H_{3} \Gamma_{C}$ is an iso- 
morphism. It follows that $j_{1}: H_{3} \Gamma_{C} \rightarrow H_{3} \Gamma_{B}$ is zero, because $0=i_{\star}=j_{1} \circ \bar{\eta}$. Summarizing, we have: $j_{1}=0, H_{3} \Gamma_{C}=H_{3} C=F, H_{3} \Gamma_{\partial K}=H_{3}(\partial K)=F$.

Part 3. Our next goal is to determine the group $H_{3} \Gamma_{K}$. Note that $K=$ $L \cup N$, where

$$
\begin{aligned}
L & =\left\{\frac{1}{2} x+r \cdot \frac{1}{4} \varphi_{x}(y):(x, y) \in S^{1} \times S^{2}, 0 \leq r \leq 1 / 2\right\}, \\
N & =\left\{\frac{1}{2} x+r \cdot \frac{1}{4} \varphi_{x}(y):(x, y) \in S^{1} \times S^{2}, 1 / 2 \leq r \leq 1\right\}
\end{aligned}
$$

and $L \cap N=\partial(L)$. For abbreviation, we let

$$
\Omega=\left(\frac{1}{2} x+r \cdot \frac{1}{4} \varphi_{x}(y), r(1-r) z\right)
$$

for $z \in q^{-1}(y)$. The homotopy

$$
G_{t}(\Omega)=\left(\frac{1}{2} x+[t+(1-t) r] \cdot \frac{1}{4} \varphi_{x}(y),[t+(1-t) r][1-t-(1-t) r] z\right)
$$

shows that $\Gamma_{\partial K}$ is a strong deformation retract of $\Gamma_{N}$. Another homotopy

$$
H_{t}(\Omega)=\left(\frac{1}{2} x+t r \cdot \frac{1}{4} \varphi_{x}(y), \operatorname{tr}(1-t r) z\right)
$$

with $H_{0}(\Omega)=\left(\frac{1}{2} x, 0\right)$ gives $\Gamma_{L} \simeq S^{1}$. We also have a homeomorphism $h: \Gamma_{\partial L} \rightarrow S^{1} \times S^{3}$ which sends $\left(\frac{1}{2} x+\frac{1}{8} \varphi_{x}(y), \frac{1}{4} z\right)$ to $(x, z)$ for $z \in q^{-1}(y)$. Consider the segment of the Mayer-Vietoris exact sequence:

$$
H_{3} \Gamma_{\partial L} \stackrel{\lambda}{\rightarrow} H_{3} \Gamma_{L} \oplus H_{3} \Gamma_{N} \stackrel{\psi}{\rightarrow} H_{3} \Gamma_{K} \rightarrow H_{2} \Gamma_{\partial L} .
$$

Since $H_{2} \Gamma_{\partial L}=H_{2}\left(S^{1} \times S^{3}\right)=0, \psi$ is an epimorphism. Clearly, $H_{3} \Gamma_{L}=$ $H_{3} S^{1}=0$. If $\lambda=0$ then $\psi$ is an isomorphism and $H_{3} \Gamma_{K} \cong H_{3} \Gamma_{N} \cong$ $H_{3} \Gamma_{\partial K} \cong H_{3}(\partial K)=F$. What is left is to show that $\lambda=0$ or equivalently, that the inclusion $\omega: \Gamma_{\partial L} \rightarrow \Gamma_{N}$ induces the zero homomorphism on $\mathrm{H}_{3^{-}}$ groups. This is equivalent to $0=\xi_{\star}: H_{3}\left(S^{1} \times S^{3}\right) \rightarrow H_{3}\left(S^{1} \times S^{2}\right)$ for $\xi=\varphi^{-1} \circ G_{1} \circ \omega \circ h^{-1}$ where $G_{1}\left(\Gamma_{N}\right)=\Gamma_{\partial K}=\partial(K) \times 0$ is identified with $\partial(K)=\varphi\left(S^{1} \times S^{2}\right)$. It is easy to check that $\xi(x, z)=(x, q(z))$. Thus $\xi=\mathrm{id} \times q$. By the Künneth theorem, the diagram

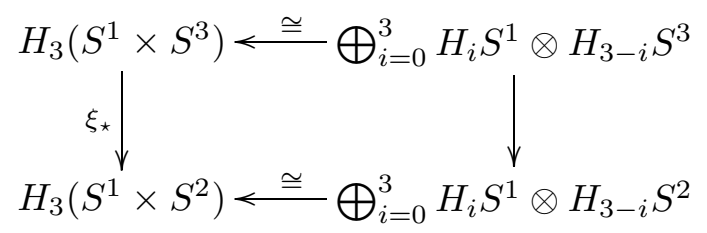

commutes. The $i$ th component of the direct sum is nonzero only for $i=0$ in the first row and only for $i=1$ in the second row of the above diagram. Hence $\xi_{\star}=0$. 
Part 4. Consider the segment of the Mayer-Vietoris exact sequence:

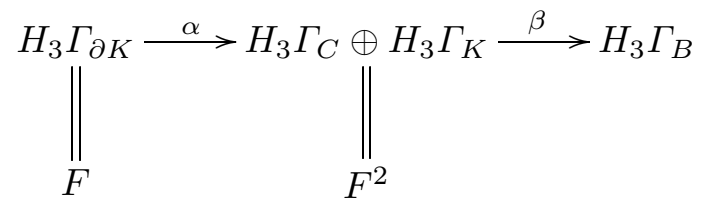

where $\alpha(x)=\left(i_{1} x, i_{2} x\right)$ and $\beta(x, y)=j_{2} y-j_{1} x=j_{2} y$ (see Part 2). Since $i_{2}$ is a composition

$$
H_{3} \Gamma_{\partial K} \stackrel{\cong}{\rightrightarrows} H_{3} \Gamma_{N} \stackrel{\psi}{\rightarrow} H_{3} \Gamma_{K},
$$

$i_{2}$ is an isomorphism (see Part 3). Now, $\operatorname{dim} \operatorname{im} \alpha=1=\operatorname{dim} \operatorname{ker} \beta$. Thus $\operatorname{dimim} j_{2}=\operatorname{dimim} \beta=2-\operatorname{dim} \operatorname{ker} \beta=1$. But $0=\beta \circ \alpha=j_{2} \circ i_{2}$. Therefore $j_{2}=0$, a contradiction.

\section{References}

[1] K. Borsuk, On some metrization of the hyperspace of compact sets, Fund. Math. 41 (1954), 168-202.

[2] T. A. Chapman and S. Ferry, Approximating homotopy equivalences by homeomorphisms, Amer. J. Math. 101 (1979), 583-607.

[3] - - - Hurewicz fiber maps with ANR fibers, Topology 16 (1977), 131-143.

[4] A. Dawidowicz, Méthodes homologiques dans la théorie des applications et des champs de vecteurs sphériques dans les espaces de Banach, Dissertationes Math. 326 (1993).

[5] A. Dold, Lectures on Algebraic Topology, Springer, Berlin, 1972.

[6] E. Dyer and M. E. Hamstrom, Completely regular mappings, Fund. Math. 45 (1957), 103-118.

[7] R. D. Edwards and R. C. Kirby, Deformations of spaces of imbeddings, Ann. of Math. 93 (1971), 63-88.

[8] S. Eilenberg and D. Montgomery, Fixed point theorems for multivalued transformations, Amer. J. Math. 58 (1946), 214-222.

[9] S. Ferry, Homotoping ع-maps to homeomorphisms, ibid. 101 (1979), 567-582.

[10] R. Fintushel, Local $S^{1}$ actions on 3-manifolds, Pacific J. Math. 66 (1976), 111-118.

[11] L. Górniewicz, Homological methods in fixed point theory of multi-valued maps, Dissertationes Math. 129 (1976).

[12] - Fixed point theorems for multivalued maps of subsets of Euclidean spaces, Bull. Acad. Polon. Sci. Sér. Sci. Math. 27 (1979), 111-115.

[13] - Present state of the Brouwer fixed point theorem for multivalued mappings, Ann. Sci. Math. Québec 22 (1998), 169-179.

[14] A. E. Hatcher, A proof of the Smale Conjecture, Diff $\left(S^{3}\right) \simeq O(4)$, Ann. of Math. 117 (1983), 553-607.

[15] D. Husemoller, Fibre Bundles, McGraw-Hill, 1966.

[16] W. Jakobsche, Approximating homotopy equivalences of surfaces by homeomorphisms, Fund. Math. 118 (1983), 1-9.

[17] -, Approximating homotopy equivalences of 3-manifolds by homeomorphisms, ibid. 130 (1988), 157-168. 
[18] J. Jezierski, An example of finitely-valued fixed point free map, Zeszyty Nauk. Wydz. Mat. Fiz. Chem. Uniw. Gdańskiego 6 (1987).

[19] H. Kneser, Die Deformationssätze der einfach zusamenhängenden Flächen, Math. Z. 25 (1926), 362-372.

[20] D. Miklaszewski, On the Brouwer fixed point theorem, Topology Appl. 119 (2002), 53-64.

[21] —, A fixed point theorem for multivalued mappings with nonacyclic values, Topol. Methods Nonlinear Anal. 17 (2001), 125-131.

[22] D. Miklaszewski, A reduction of the Nielsen fixed point theorem for symmetric product maps to the Lefschetz theorem, Fund. Math. 135 (1990), 175-176.

[23] B. O'Neill, A fixed point theorem for multivalued functions, Duke Math. J. 14 (1947), 689-693.

[24] P. Orlik and F. Raymond, On 3-manifolds with local SO(2) action, Quart. J. Math. Oxford 20 (1969), 143-160.

[25] H. Schirmer, A fixed point index for bimaps, Fund. Math. 134 (1990), 91-102.

[26] - , The least number of fixed points of bimaps, ibid. 137 (1990), 1-8.

[27] H. Seifert, Topologie dreidimensionaler gefaserter Räume, Acta Math. 60 (1933), $147-238$.

[28] S. Smale, Diffeomorphisms of the 2-sphere, Proc. Amer. Math. Soc. 10 (1959), 621-626.

[29] N. E. Steenrod and D. B. A. Epstein, Cohomology Operations, Princeton Univ. Press, 1962.

[30] R. M. Switzer, Algebraic Topology-Homotopy and Homology, Springer, 1975.

Faculty of Mathematics and Computer Science

Nicholas Copernicus University

Chopina 12/18

87-100 Torun, Poland

E-mail: miklasze@mat.uni.torun.pl

Received 31 July 2001;

in revised form 26 June 2002 